\title{
Die Handlungsfähigkeit des Nationalstaats bei der Regulierung der Finanzmärkte
}

\author{
Renate Mayntz
}

\begin{abstract}
Zusammenfassung: In der Diskussion um die erodierende Handlungsfähigkeit des Nationalstaats geht es normalerweise um Innenpolitik. Durch sein zunächst effektives Management der sich als Bankenkrise äußernden Finanzmarktkrise hat der Nationalstaat unzweifelhaft Handlungsfähigkeit bewiesen. Für die Verhinderung künftiger Krisen dieser Art ist eine striktere Regulierung der Finanzmärkte erforderlich. Wirksame Regulierung ist angesichts der globalen Expansion der Finanzmärkte nur durch internationale Vereinbarungen zu erreichen. In den entsprechenden Verhandlungen äußert sich jedoch die Handlungsfähigkeit der Nationalstaaten vor allem in der Verhinderung internationaler Vereinbarungen, die ihren nationalen Interessen nicht gerecht werden. Hier wird nationale Handlungsfähigkeit kontraproduktiv.
\end{abstract}

Schlüsselwörter: Finanzmarkt · Regulierung · Staatliche Handlungsfähigkeit

\section{The capacity of nation-states to regulate financial markets}

\begin{abstract}
The effective management of the financial crisis by national governments seems to contradict the popular diagnosis of the erosion of the national state. To avoid future crises, regulatory reform is needed. In view of the global expansion of financial markets, effective regulation requires the imposition of international norms and standards. However, Western nation states are proving unable to reach consensus on crucial measures. Their governing capacity now manifests itself mainly in obstructing internationally agreed interventions. If manifested defensively rather than proactively, national sovereignty becomes dysfunctional where problems can only be solved by international cooperation.
\end{abstract}

Keywords: Regulation $\cdot$ Financial markets $\cdot$ Governance capacity

\section{MPIfG Journal Article}

Renate Mayntz: Die Handlungsfähigkeit des Nationalstaats bei der Regulierung der Finanzmärkte. In: Leviathan 38(2), 175 - 187 (2010). Nomos

The original publication is available at the publisher's web site: http://dx.doi.org/10.1007/s11578-010-0078-6

The MPIfG Journal Articles series features articles by MPIfG researchers and visiting scholars published in peer-reviewed journals. Max Planck Institute for the Study of Societies (MPIfG) Cologne | www.mpifg.de

\begin{tabular}{l}
\hline C VS-Verlag 2010 \\
Prof. Dr. R. Mayntz ( $₫)$ \\
Max-Planck-Institut für Gesellschaftsforschung, Paulstr. 3, \\
50676 Köln, Deutschland \\
E-Mail: Mayntz@mpifg.de
\end{tabular}


Die politischen Akteure der grossen Nationalstaaten haben durch ihr teilweise in internationalen Gremien abgestimmtes Handeln bislang einen gesamtwirtschaftlichen Zusammenbruch verhindert. Die nach dem Ausbruch der Krise für kurze Zeit dominierende Rolle der Staaten gegenüber einer vor dem Zusammenbruch stehenden kapitalistischen Wirtschaft haben zu Spekulationen über eine langfristige Rückkehr des Staates als wirtschaftliches Steuerungszentrum geführt (James 2009). Erfolgreiches Krisenmanagement bedeutet aber nicht unbedingt eine „Rückkehr“ des Staates.

Der Nationalstaat hat sich, wie Schuppert (2009, S. 62) meint, in der Ausnahmesituation des Finanznotstands der (immer noch) nur ihm zustehenden Handlungsmöglichkeiten bedient: er kann, so Schuppert, nahezu unbegrenzt Geldmengen zur Verfügung stellen „und sogar anordnen, dass der Schutzschirm auch in Anspruch genommen wird“. Aber auch Staaten können, woran uns ein Blick in die Geschichte erinnert, insolvent werden (Reinhart u. Rogoff 2009). Die innenpolitische Handlungsfähigkeit hoch verschuldeter Staaten ist stark begrenzt, denn der Sparzwang, der aus der Notwendigkeit des Schuldenabbaus resultiert, schränkt fiskalrelevante politische Entscheidungen ein. So wird etwa im deutschen Fall, wie Wolfgang Streeck im vorangehenden Beitrag zeigt, durch die Ausgaben für das staatliche Krisenmanagement ein langfristiger Trend wachsender Staatsverschuldung verstärkt, denn die staatlichen Mittel müssen zunehmend zur Abgeltung ererbter Verpflichtungen eingesetzt werden.

Nicht jede Politik jedoch ist mit Ausgaben verbunden. Staatliche Handlungsfähigkeit erweist sich auch in der Fähigkeit zu gesetzlicher Normierung, die keine Leistungsansprüche begründet: Die Regulierung der Finanzmärkte verursacht dem Staat keine unmittelbaren Kosten. Die jüngste Finanzkrise wird nicht zuletzt als Folge unzureichender Regulierung, der ,gaps and inconsistencies in regulatory regimes“ der globalisierten und immer komplexer gewordenen Finanzmärkte angesehen (Draghi 2008). Um eine Wiederholung der Krise zu verhindern wird deshalb eine Reform der Finanzmarktregulierung gefordert. Wie aber steht es um die Handlungsfähigkeit der von der Krise betroffenen - und zugleich für sie mitverantwortlichen - westlichen Nationalstaaten im Hinblick auf diese Reform? Der Reformprozess ist noch in Gang, aber es zeichnen sich bereits einige Tendenzen ab, die es hier festzuhalten gilt.

\section{Zum status quo ante der Finanzmarktregulierung}

Die Krise konnte ausbrechen, weil die institutionelle Einbettung der Ökonomie offensichtlich defizitär war. Obwohl traditionell der Selbstregelung überlassen, hat die hohe, nicht nur wirtschaftliche sondern auch politische Bedeutung des Finanzsystems die Politik seit der frühen Neuzeit immer wieder veranlasst, auf nationaler Ebene regelnd ins Bankensystem und den Kapitalverkehr einzugreifen. ${ }^{1}$ Immer wieder waren es dabei Krisen, die zu politischer Intervention führten; ein Beispiel ist der Erlass des Glass-Steagall Gesetzes in den USA, mit dem die Politik 1933 auf eine der vermuteten Ursachen der damaligen Krise, die zunehmenden Investmenttätigkeiten von Geschäftsbanken, reagierte. Als der Zweite Weltkrieg heraufzog, wurden Zinsen, Wechselkurse und der Kapitalverkehr verschärft

1 Zum Folgenden die Beiträge in Mayes u. Wood 2007. 
reguliert. Nach dem Zweiten Weltkrieg ging es zunächst um den Wiederaufbau; seit dem Bretton Woods-Abkommen (1944) galten feste Wechselkurse, und ein kooperatives Verhältnis zwischen Banken, Großunternehmen und Politik bot wenig Anlass zu verschärfter nationaler Regulierung. Das änderte sich, als zu Beginn der 1970 Jahre das BrettonWoods-System fester Wechselkurse in der westlichen Welt zerbrach. Damit gewann nicht nur der Devisenmarkt an Bedeutung; die westlichen Nationalstaaten begannen zugleich, den Kapitalmarkt zu liberalisieren (vgl. u. a. Underhill 1991). Freie Wechselkurse und die Abschaffung von Kapitalverkehrskontrollen ermöglichten die Entstehung internationaler Finanzmärkte rechtlich; zugleich machte die Entwicklung moderner Informations- und Kommunikationstechnik sie praktisch möglich. Besonders aktiv bei der Deregulierung waren zunächst die USA und später Großbritannien. In den USA erlaubte zum Beispiel der 1970 novellierte Bank Holding Company Act die Bildung von komplex strukturierten Bank-Holding Companies; in Großbritannien wurde 1971 die Regulierung der Kreditvergabe abgeschafft. Die Europäische Union verfolgte, ihrem Mandat getreu, die Schaffung eines integrierten europäischen Finanzmarkts. ${ }^{2}$ Die 1994 in der Uruguay-Runde generell vereinbarte Öffnung nationaler Märkte für finanzielle Dienstleistungen trieben die Internationalisierung von Banken und Versicherungsunternehmen weiter voran und die im Jahr 2000 auf europäischer Ebene im Financial Services Action Plan zusammengefassten Maßnahmen und Richtlinien dienten in erster Linie diesem übergeordneten Ziel. Auch die europäische Rechtssetzung in diesem Gebiet bedeutete Liberalisierung, nicht restriktive Regulierung. Nach Auffassung des seinerzeit zuständigen Europäischen Kommissars McCreevy brachte die wichtige Markets in Financial Instruments Directive denn auch nicht mehr, sondern weniger Regulierung (Economist 9. September 2006). Das u.a. durch die Währungsfluktuation bedingte Risiko bei internationalen Geschäften führte folgerichtig zum Wunsch nach Absicherung, was die Einführung neuer Finanzprodukte begünstigte. Immer komplexer strukturierte Derivate wurden außerbörslich gehandelt; in Deutschland wurde die Regelung von Derivaten zwischen 2002 und 2005 aus dem Börsengesetz getilgt und in das Wertpapierhandelsgesetz eingeschrieben und damit umfassend dereguliert (Reifner 2010, S. 313-315). In bestimmten deregulierten Zonen konnten Banken sogenannte Zweckgesellschaften gründen, mit deren Hilfe sie ihre Bilanzen schönten.

Die komplexer werdenden Finanzmärkte verlangten nach stärkerer Aufsicht. Auf nationaler Ebene waren dafür die Zentralbanken, Finanzministerien und eine Reihe spezialisierter Aufsichtsinstanzen zuständig. Die Börsenaufsicht wurde auf nationaler Ebene zentralisiert (Deeg u. Lütz 2000), und in Ländern wie Großbritannien und Deutschland wurde die Aufsicht über Banken, Wertpapierhandel und Versicherungen in einer Behörde zusammengefasst. In Großbritannien entstand so 1997 die Financial Services Agency FSA, in Deutschland 2002 die Bundesanstalt für Finanzdienstaufsicht BaFin. ${ }^{3}$ In vielen entwickelten kapitalistischen Ländern gibt es neben einem Finanzministerium und einer Zentralbank spezielle Aufsichtsinstanzen für Banken, Wertpapierhandel und das

2 Zum Folgenden vgl. Quaglia 2007; de Larosière 2009.

3 Das in der BaFin aufgegangene Bundesaufsichtsamt für das Kreditwesen war 1962 geschaffen worden. 
Versicherungswesen. ${ }^{4}$ In dem pluralisierten System der USA, wo selbst die Zentralbank zwei Ebenen (federal und state) hat, existieren für verschiedene Finanzmärkte verschiedene Aufsichtsbehörden.

Die Internationalisierung der Finanzmärkte führte aber nicht nur reaktiv zu Veränderungen in der nationalen Regulierung; sie wurde auch vom schrittweisen Entstehen internationaler Regulierungsinstitutionen begleitet. ${ }^{5}$ Damit reagierte man auf den mit der Liberalisierung und Internationalisierung erkennbar gewachsenen Koordinationsbedarf. So entstand 1974 aus der Kooperation von Notenbankgouverneuren und nationalen Behörden für die Bankenaufsicht das an die Bank für Internationalen Zahlungsausgleich (BIZ) angelagerte Basler Komitee, das Regeln für die Kooperation von nationalen Aufsichtsbehörden und für die Eigenkapitalunterlegung der Banken entwickelte. 1983 entstand die IOSCO als internationale Vereinigung der Wertpapieraufsichtsbehörden, 1996 wurde die International Association of Insurance Supervisors (IAIS) gegründet, und 2001 entstand aus einem seit 1973 bestehenden internationalen Ausschuß das International Accounting Standards Board (IASB). Ähnliche Gremien wurden im Rahmen der EU etabliert. Im Jahr 1999 schließlich wurde nicht nur das internationale Forum für Finanzstabilität (FSF) gegründet, das die Entwicklung von Standards für die Beaufsichtigung von Banken, Versicherungen und Wertpapiermärkten koordinieren sollte; die Finanzminister der G7, die sich seit Mitte der 90er Jahre zunehmend mit Fragen der Finanzaufsicht beschäftigt hatten, schufen außerdem die G20 als Forum, in dem Finanzminister und Zentralbankgouverneure sich gemeinsam um die Stabilität des internationalen Finanzsystems bemühen sollten. ${ }^{6}$ Bekanntlich ließen es sich später die Regierungschefs nicht nehmen, selbst zu den G20-Treffen zu kommen.

Aus den verschiedenen separaten Initiativen entstand keine internationale Finanzmarktregelung aus einem Guß, sondern ein eher fragmentiertes als kohärentes Geflecht an Institutionen. Anders als im Fall der WTO haben die einzelnen Staaten keine Regelungskompetenzen an die neu entstandenen internationalen Institutionen der Finanzmarktregelung abgetreten, geschweige denn eine Instanz geschaffen, die imstande gewesen wäre, eine der wachsenden Expansion und Komplexität der Finanzmärkte angepasste internationale Finanzmarktordnung zu errichten. Die meisten Institutionen der internationalen Finanzmarktregelung haben den Charakter von Verhandlungssystemen, in denen eine kleine Zahl nationaler Repräsentanten um die Formulierung von Standards ringen und sich auf dem Niveau des kleinsten gemeinsamen Nenners einigen. Nationale Repräsentanten sind vor allem Finanzminister und Vertreter von Zentralbanken und/oder nationalen Aufsichtsbehörden, manchmal ergänzt um weitere Experten und um Vertreter von Regelungsadressaten. ${ }^{7}$ Die von den genannten internationalen Institutionen entwickelten Regeln und Standards haben nur Empfehlungscharakter und werden von einzelnen Regierungen ebenso wie von Banken und Investoren vielfach missachtet oder lediglich

4 Braithwaite u. Drahos 2000, Kap. 8; Busch 2009; Padoa-Schioppa 2003.

5 Zum Folgenden vgl. auch Lütz 2009.

6 Ebenfalls 1999 wurde im Internationalen Währungsfonds das International Financial and Monetary Committee IFMC etabliert.

7 Auf internationaler Ebene ist von den hier genannten Institutionen nur das International Accounting Standards Board ein reines Expertengremium. 
formal akzeptiert, aber faktisch unterlaufen. Das Schwergewicht der bisherigen Finanzmarktregulierung lag damit selbst dort, wo internationale Standards entwickelt wurden, eindeutig bei den nationalen Akteuren; durch das Entstehen internationaler Institutionen der Finanzmarktregelung wurde die autonome Entscheidungsfähigkeit der Nationalstaaten nicht ernsthaft eingeschränkt.

Die globale Finanzkrise war denn auch vor allem eine Folge nationaler Entscheidungen und Nicht-Entscheidungen. Die Zahl verabschiedeter europäischer und internationaler Regelungen sagt wenig über die Regulierungsintensität, insbesondere dann, wenn neue Normen, wie zumeist im Fall der EU, nicht der Disziplinierung sondern der Erweiterung von Märkten dienen. Ähnliches kann man über die zunehmende Koordination nationaler Behörden sagen, die über die Einhaltung von Regeln wachen, die den Handlungsspielraum von Finanzmarktakteuren eher erweitern statt ihn einzuschränken. Banken und Investoren besitzen ein ausgeprägtes Eigeninteresse und bedienen sich hochbezahlter Spezialisten, um existierende Regulierungen (z.B. durch die Nutzung von Steueroasen und Zweckgesellschaften) zu umgehen. Nationales Recht blieb zwar entscheidend, zeigte jedoch vor allem in Hinblick auf die sogenannten „Finanzinnovationen“ der Finanzmarktakteure gravierende Regelungslücken, und nationale Aufsichtsbehörden (wie die deutsche BaFin) verfügten nur über beschränkte Eingriffsrechte, Ressourcen und Sanktionsmittel. Die an sich schon unzureichenden nationalen Regelungen wurden außerdem nicht durchgängig angewandt; das gilt speziell für Großbritannien, das in der Zeit des Börsenbooms bemüht war, seinem Finanzplatz möglichst wenig regulative Fesseln anzulegen. Die Nationalstaaten waren mit ihrer Politik verantwortlich für die Krise. Wie haben sie regelnd darauf reagiert?

\section{Nationale Reaktionen auf die Krise - wirkungsvoll?}

Die Krise hat zunächst dazu geführt, dass bestehende Regulierungsinstanzen ihre Kompetenzen stärker ausschöpfen und Regeln strikter anwenden. Die bislang so tolerante britische Aufsichtsbehörde FSA hat ein „Supervisory Enhancement Program“ (SEP) entwickelt, und auch die Aufsichtsbehörden in Frankreich und Deutschland ziehen die Zügel an. In den USA ging die Aufsichtsbehörde SEC gegen den Insiderhandel vor, und Manager von Hedgefonds, die mit ihren Spekulationen die Krise mit verursacht haben, wurden vor Gericht gebracht. ${ }^{8}$ Wenn es, wie das EZB Vorstandsmitglieds Lorenzo Bini Smaghi (2009) meint, nicht primär an fehlenden Instrumenten gelegen hat, dass die Finanzkrise nicht verhindert wurde, sondern daran, dass vorhandene Instrumente nicht hinreichend genutzt wurden, dann sind dies angemessene Reaktionen.

Allgemein wurde jedoch eine striktere Anwendung bestehender Regeln nicht als ausreichend erachtet, künftige Finanzkrisen zu verhindern. Vielmehr forderten Politiker zumal in den Ländern, die mit ihrem bisherigen Verhalten im Zentrum der Kritik standen, mit starken Worten eine neue und striktere Regulierung. In Großbritannien machte die Turner Review der Aufsichtsbehörde FSA im März 2009 umfassende Reformvorschläge (FSA

8 Vgl. Financial Times (FT) 7.8.2009; Financial Times Deutschland (FTD) 22.11.2009; FTD 24.10.2009, FT 12.11.2009; FTD 9.10.2009. 
2009), im November kündigte die Queen ein neues Gesetz über Finanzdienstleistungen (fiscal services bill) an, und Schatzkanzler Darling machte sich für die Empfehlungen des Walker-Berichts stark, bei dem es um die Reform der Gehaltszahlung an Bankmitarbeiter geht. In den USA präsentierte Präsident Obama im Sommer 2009 ein umfassendes Paket von Maßnahmen, durch die nicht nur die Regulierungskompetenzen der amerikanischen Notenbank (Federal Reserve) erweitert, sondern auch eine neue Institution für den Schutz der Nutzer von Finanzdienstleistungen geschaffen würde (Department of the Treasury 2009). Adressaten der auf dem Höhepunkt der Krise verlangten Reformen sind vor allem Banken, aber auch Hedgefonds, Ratingagenturen und Steueroasen. Von den Banken wird ein Bezahlungssystem für Mitarbeiter und Manager verlangt, das keinen Anreiz zu riskanten Entscheidungen bietet, außerdem eine größere Eigenkapitalunterlegung, eine bessere Liquiditätsvorsorge und ein besseres Risikomanagement; verschiedentlich wird gefordert, die Größe vor allem von international tätigen Banken zu beschränken und sie zu zwingen, Vorkehrungen für eine geordnete Insolvenz zu treffen, um Kettenreaktionen wie im Fall von Lehman Brothers zu verhindern.

Den umfassenden Reformforderungen folgten einzelne Handlungen nationaler Regierungen und Zentralbanken. So veränderte etwa die spanische Zentralbank im Juli 2009 die Bilanzierungsvorschriften für Banken, Großbritannien führte strengere Regeln für die Liquidität von Banken ein, in den Niederlanden wurden die Gehälter von Bankmanagern beschränkt, in den USA wurden staatliche Kredite für Banken an Gehaltsobergrenzen oder Beschränkungen bei Bonuszahlungen gekoppelt, Schweden führte eine Stabilitätssteuer für Banken ein, Großbritannien verlangte als erstes Land eine strengere Liquiditätsvorsorge von Banken, und im Dezember 2009 entschieden sich Großbritannien und Frankreich, die Boni von Bankern einmalig zu50\%zu besteuern. ${ }^{9}$ Der Schwerpunkt nationaler Aktivität lag jedoch im Krisenjahr 2009 bei Versuchen, ein funktionsfähiges Bankensystem zu erhalten und der auf die Finanzmarktkrise folgenden Rezession entgegen zu steuern. Auch jenseits von Liquiditätshilfen, Verstaatlichung insolventer Finanzinstitute und Konjunkturprogrammen haben nationale Regelungen wie die Zulassung von „Bad Banks" primär dem Krisenmanagement gedient.

Die Bedeutung nationaler Initiativen zur Verbesserung der Finanzmarktregulierung ist schwer zu beurteilen. Wenn die Krise nicht zwangsläufig aus der Beschaffenheit der internationalen Finanzmärkte entstand, sondern durch das auf private und öffentliche Verschuldung zurückgehende und mit einem massiven Zahlungsbilanzdefizit erkaufte Wachstum in den USA, also durch ein einzelnes Land ausgelöst wurde, dann mag in der Tat entscheidend sein, wie nationale Regierungen die auf den nationalen Märkten tätigen Finanzmarktakteure regulieren. Die britische Warwick Commission spricht sich denn auch dezidiert für eine vorrangig nationale Bankenregulierung aus: ,We suggest that national regulatory autonomy goes hand in hand with legitimate international cooperation on a wide range of issues" (The Warwick Commission 2009, S. 6). Nationale Reformansätze sind jedoch landesintern zumeist umstritten. Obamas weitreichende Reformvorschläge stießen von Anfang an nicht nur bei Republikanern, sondern auch in seiner eigenen Partei und bei den betroffenen Aufsichtsinstitutionen auf hartnäckige Kritik, und

9 FT 17.7.2009, 9.9.2009, 6.10.2009, 10.12.2009. 
auch in Großbritannien wurde die Absicht einer schärferen Regulierung zum Gegenstand der Auseinandersetzung zwischen Regierung und Opposition.

Selbst wenn es keine Meinungsverschiedenheiten zwischen politischen Akteuren gibt die Fähigkeit nationaler Regierungen zur effektiven Finanzmarktregelung ist beschränkt. Zum einen treffen Versuche strikterer Regulierung auf nationaler Ebene auf den entschiedenen Widerstand verschiedener Gruppen von Finanzmarktakteuren, die sich mit ihren nach wie vor mächtigen Lobbies gegen Regeln wehren, die ihre Handlungsspielräume und damit ihren Profit einschränken. Alleingänge etwa bei der Beschränkung von Boni oder der Erhöhung der Eigenkapitalanforderungen, so die Furcht, könnten nationale Finanzmarktakteure im internationalen Wettbewerb benachteiligen. Zum anderen sind die Möglichkeiten nationaler politischer Akteure beschränkt, regelnd auf die Finanzmarktakteure einzuwirken. Ganz allgemein ist die rechtliche und praktische Möglichkeit des Zugriffs auf diejenigen Akteure, deren Verhalten politisch reguliert werden soll, die zentrale Voraussetzung nationalstaatlicher Handlungsfähigkeit (Mayntz 2009). Diesem Zugriff aber sind durch die internationale Ausbreitung und die Verflochtenheit der Finanzmärkte und den zunehmend multinationalen Charakter großer Banken und Finanzholdings enge Grenzen gesetzt. Schon vor zwanzig Jahren hat Susan Strange (1988) das globalisierte Finanzsystem selbst, und nicht etwa das Entstehen internationaler Regulierungsinstitutionen als Ursache der von ihr diagnostizierten Erosion nationalstaatlicher Handlungsfähigkeit identifiziert. Deshalb ist selbst in Ländern, deren Regierungschefs oder Finanzpolitiker zunächst mit umfassenden Reformvorschlägen auf die Krise reagiert haben, bisher tatsächlich nicht wirklich viel geschehen.

\section{Der Nationalstaat als Akteur im internationalen Reformprozess}

War Krisenmanagement eindeutig eine nationale Angelegenheit, dann betonten nicht nur außenstehende Beobachter, sondern auch nationale Politiker von Anfang an, dass Reformen, die allein auf die je eigenen, nationalen Finanzunternehmen gerichtet sind, angesichts der globalen Ausdehnung und hohen internationalen Verflechtung der Finanzmärkte nicht ausreichen. Wenn ein Land allein, und seien es die USA, z. B. Credit Default Swaps verbieten würde, dann würden diese Instrumente dadurch noch keineswegs von der globalen Bildfläche verschwinden. Einigkeit bestand deshalb sehr schnell darüber, dass isolierte nationale Reformen wenig sinnvoll sind. Tatsächlich zeigt eine Durchsicht der in der britischen Turner Review aufgelisteten Reformempfehlungen (Financial Services Agency 2009, S. 118-122), dass fast alle Reformvorhaben, die sich auf Standards und das Verhalten international tätiger Finanzinstitute beziehen, eine internationale Vereinbarung erfordern. Das Leitmotiv der letzten Monate, so Lorenzo Bini Smaghi (2009), war daher: „if the crisis is global, the solution must also be global“. Da jedoch keine genuin supranationalen Regelungsinstitutionen für den Finanzmarkt existieren, hängt eine wirksame Reform der Finanzmarktregulierung von der Kooperation zwischen nationalen Regierungen ab - gleichgültig, ob es um die Harmonisierung der verschiedenen nationalen Regelungen oder um Veränderungen an der internationalen Regulierungsstruktur geht. 
Betrachtet man allerdings, wie im Laufe des Jahres 2009 auf der Ebene der G20 und internationaler Institutionen auf die Krise reagiert wurde, dann wird deutlich, dass, wie auf der nationalen Ebene, die in die Wege geleiteten und die noch geplanten Maßnahmen vordringlich der Krisenbewältigung durch Stützung des Bankensystems und der Konjunktur dienen. Die Bilanz echter regulatorischer Reformen war dagegen Ende 2009 noch mager. Neue Regulierungsinstanzen wurden auf internationaler Ebene nicht geschaffen; stattdessen wurden bestehende Institutionen ausgebaut. Die G20 avancierten zum wichtigsten Forum nicht nur gemeinsamer Krisenbekämpfung, sondern auch internationaler Reformbestrebungen. Das bisherige Financial Stability Forum FSF wurde um die Vertreter von Schwellenländern erweitert und zum Financial Stability Board FSB ausgebaut, das als wichtigstes Instrument der G20 fungiert (Arner u. Taylor 2009). Auch das Basler Komitee wurde um Vertreter von Schwellenländern erweitert, und innerhalb des IMF soll sich nach dem Willen der G20 die Stimmrechtsverteilung bis 2011 ebenfalls ändern. Damit soll der in den letzten Dekaden gewachsenen ökonomischen Bedeutung der Schwellenländer Rechnung getragen werden, die mit in die Verantwortung etwa für die Probleme globaler Handelsbilanzungleichgewichte gezogen werden sollen. Was Regulierungsreformen angeht, liegt das Schwergewicht der von den G20 formulierten, selten über allgemeine Richtungsempfehlungen hinausgehenden Desiderate bei verbesserter Kooperation von nationalen und von internationalen Institutionen. Das FSB soll zwischen dem Internationalen Währungsfonds und anderen Regulierungsinstitutionen vermitteln, und bei der Aufsicht über die großen international tätigen Finanzinstitute sollen die nationalen Aufsichtsinstanzen künftig in einem entsprechend zusammengesetzten college of supervisors zusammenwirken (G20 2009).

Die Regierungen, Notenbanken und Aufsichtsinstitutionen verschiedener Länder können sich jedoch nur schwer auf ein gemeinsames Vorgehen einigen. Das politische Interesse an einer schärferen Regulierung ist generell begrenzt; schärfere Regulierung kann höhere Zinsen und niedrigeres Wachstum bedeuten, was beides dazu führt, dass die Staatsverschuldung noch schwieriger abzubauen ist. Auch die Bedeutung des nationalen Finanzsystems für das Funktionieren der nationalen Wirtschaft schützt es vor radikalen Eingriffen der Politik, die Angst davor hat, es lahmzulegen. Internationale Vereinbarungen werden außerdem durch die Verschiedenheit der nationalen Interessen erschwert. Während Staaten wie Großbritannien und die USA, die einen guten Teil ihrer Steuereinkünfte aus der Finanzindustrie bezogen haben, die eigenen Banken und den in der Periode der Finanzmarktliberalisierung entstandenen internationalen Vorrangstatus des eigenen Finanzplatzes schützen wollen, hat das vom Export abhängige Deutschland vor allem Interesse an einem erneuten Anwachsen der Güternachfrage. Meinungsverschiedenheiten gibt es nicht nur innerhalb der verschiedenen internationalen Gremien; auch in der EU treffen die Reformvorschläge der Kommission bei einzelnen Ländern auf Kritik oder Widerstand. Die jeweiligen nationalen Interessen werden durch die aktuelle Position eines Landes im Geflecht internationaler politischer und der damit verbundenen weltwirtschaftlichen Strukturen mitbestimmt und sind deshalb nur beschränkt verhandelbar. Gerade weil sie ihre Handlungsfähigkeit für die Verfolgung nationaler Interessen bewahren wollen, können die Staaten sich nicht auf Maßnahmen einigen, die nicht mindestens pareto-optimal sind. 
Auch Regulierungsbetroffene wehren sich gegen europäische und internationale Reformpläne. Die Bankenlobby in Brüssel, Berlin und London hat sich mit mindestens aufschiebender Wirkung z. B. gegen die Einführung einer Verschuldungshöchstgrenze für Banken in der EU wie auch gegen schärfere Prüfregeln für den Derivatehandel gewehrt, wobei sie auch Unterstützung von großen Unternehmen erhielt. ${ }^{10}$ Immer wieder wird dabei die Kritik an der geplanten Regulierung nicht nur mit den Folgekosten für die Banken, sondern mit ihrer angeblich zu erwartenden oder doch möglichen wachstumshemmenden Wirkung begründet. Abgesehen von handfesten Interessen beeinträchtigt schließlich auch kognitive Unsicherheit die Meinungsbildung über die notwendigen Reformen. Die Experten sind sich nicht einig, wie ein neues Regime aussehen sollte. Die einen verlangen eine strikte Regulierung auf nationaler und internationaler Ebene, die Abschaffung von Steueroasen, eine strenge Kontrolle von Hedgefonds, das Verbot einer Reihe von Finanzinnovationen wie intransparenten Verbriefungen und eine „Weltwirtschaftsregierung“. Andere Experten weisen dagegen darauf hin, dass eine funktionierende kapitalistische Wirtschaft Kredit braucht, das Geschäft mit Krediten unvermeidlich riskant ist und etwa Steueroasen Jahrzehnte lang auch positive, wenn auch wenig beachtete ökonomische Funktionen erfüllt haben. Kognitive Unsicherheit und die unter Mitwirkung der Betroffenen definierten nationalen Interessen zusammen behindern bisher die Einigung auf radikale Reformen der internationalen Finanzmarktregulierung.

Angesichts dieser Widerstände und Unsicherheiten überrascht es nicht, dass von den zunächst angemahnten Reformen der Finanzmarktregulierung auf internationaler Ebene bis Ende 2009 wenig umgesetzt wurde. Vordergründig erfolgreich scheint vor allem der Kampf gegen Steueroasen zu sein; nachhaltig von Frankreich und Deutschland unterstützt, haben neunzehn OECD Länder am 23.6.2009 ein gemeinsames Vorgehen gegen Steueroasen verabredet. Sowohl in Verhandlungen im Rahmen der OECD wie in bilateralen Vereinbarungen haben sich inzwischen mehrere Länder bereit erklärt, strengere Regeln zur Verhinderung von Steuerflucht auf dem Weg über Steueroasen zu befolgen. Da aber Auskunftspflichten der Banken in diesen Ländern an die Existenz eines konkreten Anfangsverdachts gebunden sind, dürfte die tatsächliche Wirkung der Vereinbarungen gering sein - deutlich geringer als die Wirkung einer CD mit Bankkundendaten, die in die Hände von Aufsichtsbehörden bzw. Finanzämtern gelangen. Was die Bankenregulierung angeht, hat das Baseler Komitee BCBS zwar im Juli 2009 eine neue Eigenkapitalregel verabschiedet, aber Ende 2009 wurde immer noch über Grundsatzfragen diskutiert, und die vorgeschlagenen neuen Regeln sollen vor ihrer geplanten Verabschiedung im Sommer 2010 erst getestet werden. Auch das IASB hat an neuen Standards für Bankbilanzen gearbeitet und im November 2009 einen Standard (IAS 39) verabschiedet, der, wie üblich, lediglich eine Empfehlung darstellt, gegen deren ungeprüfte Übernahme die EU sich bereits gewehrt hat.

Die EU hatte sich mit ihrem European Economic Recovery Plan (EERP) vor allem an die Mitgliedsländer gewandt und sie zunächst dazu angehalten, ihr konjunkturpolitisches Handeln miteinander abzustimmen. Auch der größte Teil der von der EU selbst in die Wege geleiteten Maßnahmen ist im weitesten Sinne konjunkturpolitisch begründet; hier wie bei den an zweiter Stelle stehenden fiskalischen Stützungsmaßnahmen der

10 Vgl. FTD 31.7.2009; FT 6.10.2009; FT 15.11.2009. 
europäischen Banken (European Central Bank ECB, European Investment Bank EIB und European Bank for Reconstruction and Development EBRD) geht es praktisch meist um kurzfristig mögliche Anpassungen bzw. Aufstockungen bereits existenter Programme. Einzelne über das aktuelle Krisenmanagement hinausgehende Initiativen sind allerdings bereits wirksam geworden. So verlangt die EU eine strengere Kontrolle von Ratingagenturen, was in Deutschland Ende 2009 zu einem entsprechenden Gesetzesentwurf geführt hat, und sie hat mehrere Mitgliedsländer, darunter auch Deutschland und Großbritannien gezwungen, große, in finanzielle Schwierigkeiten geratene Banken (z. B. Commerzbank, Royal Bank of Scotland) radikal umzustrukturieren. Im September 2009 schlug die Kommission schließlich die Einrichtung von drei europäischen Instanzen vor, eine European Banking Authority, eine European Insurance and Occupational Pensions Authority, und eine European Securities and Markets Authority. Die Vorschläge sollen im Sommer 2010 im Europäischen Parlament behandelt werden; schon im Vorfeld haben Deutschland und Großbritannien dagegen protestiert, dass die europäischen Aufsichtsinstanzen nicht nur überwachen und koordinieren, sondern unter bestimmten Umständen auch in die nationale Regulierung eingreifen könnten. ${ }^{11}$

Die tatsächlich verabschiedeten regulatorischen Reformen bleiben, wie Kommentatoren immer wieder unterstreichen, bislang weit hinter den umfassenden Reformvorstellungen der G20 zurück. Aber nicht nur die bisherigen Reformen hinken hinter dem zu Beginn der Krise als notwendig Erachteten hinterher; auch die Reformdiskussion scheint sich verengt zu haben. Zwar ist man sich grundsätzlich einig, dass es um Prävention, um die Verhinderung der nächsten, möglicherweise nicht mehr politisch abzufangenden Krise geht. Aber trotz der Vielschichtigkeit der Ursachen, die zu der letzten Krise beigetragen haben, hat sich die Reformdiskussion immer mehr auf das Verhalten von Bankern und von Banken, speziell von „systemrelevanten“ multi-nationalen Banken verengt. Bei vielen Reformvorschlägen geht es um die Schaffung von mehr Transparenz, etwa durch Einführung von Registrierungs- und Berichtspflichten für bisher unregulierte Institute und Finanzinstrumente. Das entspricht der weit verbreiteten Meinung, dass asymmetrische Informationsverteilung und fehlendes Wissen um die tatsächlich in vielen der gehandelten Papiere enthaltenen Verlustrisiken eine, wenn nicht die wesentliche Ursache der Krise des Finanzsystems war (vgl. Major 2009). Diese Meinung hat selbst die British Academy in ihrer Antwort auf eine Anfrage der Queen vertreten (British Academy 2009). Die Vorstellung, dass eine Erhöhung von Transparenz im System finanzieller Transaktionen, ein besseres Wissen von Bankern wie von kleinen und grossen Investoren um Risiken, eine Wiederholung der Krise verhindern würde, könnte sich jedoch am Ende als rationalistisches Vorurteil erweisen, denn das Verlangen nach mehr Transparenz basiert auf dem inzwischen desavouierten Glauben an die Kraft vollständiger Information als Basis sich selbst regelnder Märkte.

11 vgl. FTD v. 21.9.2009. 


\section{Das Paradox nationalstaatlicher Handlungsfähigkeit bei der Reform der Finanzmarktregulierung}

Bei der Reform der krisenträchtigen bisherigen Finanzmarktregulierung, durch die eine Wiederholung der jetzigen Krise vielleicht verhindert werden könnte, hat sich der Nationalstaat nur eingeschränkt handlungsfähig gezeigt. Nach innen wird sein Handlungswille durch das Interesse an Wachstum und Wettbewerbsfähigkeit, seine Handlungsfähigkeit durch den Widerstand von Regelungsbetroffenen beschränkt. Die staatliche Handlungsfähigkeit wird zugleich durch die Tatsache beschränkt, dass die Zuständigkeit des Nationalstaats für die Regelung des Finanzsektors innerhalb der verfassungsmäßig gezogenen Grenzen zwar unbestritten, jedoch angesichts des heutigen globalen Finanzsystems nur eingeschränkt wirksam ist. Nach außen dagegen, d.h. in internationalen Verhandlungen, haben einzelne Staaten ihre Handlungsfähigkeit vor allem durch Verweigerung, also defensiv bewiesen. Die unmittelbaren politischen Kosten der Verweigerung sind oder erscheinen zumindest geringer, als die politischen Kosten der Zustimmung zu internationalen Regelungen, die den kurzfristigen nationalen Interessen widersprechen. In der Diskussion um die Handlungsfähigkeit des Nationalstaats steht die innenpolitische Gestaltungsfähigkeit im Vordergrund. Handlungsfähigkeit erweist sich auch in der Abwehr von Forderungen, seien es Forderungen nationaler Interessengruppen oder Kooperationszumutungen auf europäischer bzw. internationalem Parkett. Wenn für die Verhinderung künftiger Krisen Kooperation unter Staaten notwendig ist, dann wird das, was nach innen gerichtet die Stärke eines Staates ausmacht - die Bewahrung seiner Souveränität - kontraproduktiv: Die sich in der Defensive bewährende einzelstaatliche Handlungsfähigkeit wird hier dysfunktional. Die durch die Initiativen beim Krisenmanagement wieder ins allgemeine Bewusstsein gerückte Handlungsfähigkeit des Nationalstaats kann einem erfolgreichen Bemühen im Wege stehen, die Wiederholung solcher Krisen durch internationale Regulierung zu verhindern.

Wenn Nationalstaaten fähig sind, sich wirkungsvoll gegen internationale Regulierungsinitiativen zu wehren, die im Widerspruch zu ihrem nationalen Interesse zu stehen scheinen, dann bestimmen Machtunterschiede die Beschaffenheit der internationalen Regelungsstruktur: Die internationale Machtverteilung wird entscheidend für das Ergebnis der Bemühungen um eine Reform der Finanzmarktregulierung. Nicht „der“ Nationalstaat erweist sich dann im Hinblick auf eine internationale Regulierungsreform als handlungsfähig, aber einzelne Staaten oder Staatenbünde. Gerade die USA, die sich vor anderen Staaten ein umfangreiches nationales Reformprogramm auf die Fahnen geschrieben und dabei am Ende des umfangreichen Textes vom Juni 2009 auch ein Lippenbekenntnis zur besseren internationalen Regulierung abgegeben hatten, scheinen im Guten (proaktiv) wie im Bösen (verhindernd) Reformversuche auf internationaler Ebene zu beeinflussen. Die USA, so steht zu vermuten, sind auch dafür verantwortlich, dass das Problem des massiven ökonomischen Ungleichgewichts in den Zahlungsbilanzen von USA und China bei den gegenwärtigen Reformbemühungen ausgeklammert bleibt - obwohl gerade dieses Ungleichgewicht und damit die amerikanischen Verschuldung gegenüber China als Mitursache der Krise gilt. Bezeichnenderweise wurde die Beseitigung von Handelsbilanzdefiziten bzw. - überschüssen weder in den Beschlüssen des April-Gipfels der G20 noch in der Abschlussverlautbarung ihres Treffens im September 
2009 angesprochen. Internationale Regulierung wurde schon immer von starken Kräften in den USA als Bedrohung wahrgenommen. Ob sich auf der Ebene der G20 am Ende die USA durchsetzen werden, welche Ziele sie dabei verfolgen, wie groß das Gewicht der EU sein wird und ob die zwischenzeitlich zu beobachtenden Ansätze zu transatlantischer Kooperation (Posner 2009) fortgesetzt oder abgebrochen werden lässt sich zur Zeit nicht absehen. Das Fehlen eines ernsthaften Bemühens, die Beseitigung globaler Ungleichgewichte auf die politische Tagesordnung zu setzen, unterstreicht den Anspruch der USA auf die Position der Hegemonialmacht - einer Position, die China bereits in Frage zu stellen beginnt.

Am Ende ein Wort der Vorsicht zu der in der gegenwärtigen Reformdiskussion verbreiteten Annahme, die großen Nationalstaaten - und hier wären auch die BRIC-Staaten (Brasilien, Russland, Indien, China) einzuschließen - könnten sich bei gutem Willen, wenn sie nur bereit wären, auf die engstirnige Verfolgung partikularer Interessen zu verzichten, auf eine Finanzmarktregelung einigen, die künftige Krisen der jetzt durchlittenen Art verhindert. Nicht nur zeigt die Geschichte eine chronische Wiederkehr von Bankenkrisen. Es mag in wirtschaftlich und technologisch hochentwickelten Gesellschaften auch prinzipielle Grenzen der ökonomischen Steuerung geben, die selbst ein staatssozialistisches Regime nicht überwinden kann. Schließlich lehrte schon die Theorie politischer Steuerung, dass erfolgreiche Steuerung nicht nur von der Steuerungsfähigkeit der Politik, sondern auch von der Steuerbarkeit des intendierten Regelungsgegenstands abhängt. Wieweit sind die politisch entgrenzten und auf eine hoch entwickelte Informations- und Kommunikationstechnik gestützten Finanzmärkte noch durch politische Intervention steuerbar? Nicht nur Politiker, sondern auch Investoren und sogar die Banker selbst scheinen im Fall der jüngsten Krise nicht nur unwillig, sondern vor allem auch unfähig gewesen zu sein, den genauen Umfang des Verlustrisikos zu erkennen, das in den von ihnen ge- und verkauften Papieren (speziell CDSs und CDOs) enthalten ist; sie haben, wie am Ende auch die Rating-Agenturen, den Ergebnissen mathematischer Modelle zur Risikoberechnung vertraut, die sie nicht nachvollziehen und damit auch nicht kritisch beurteilen konnten. Nach der teilweisen Automatisierung des Wertpapier- und Devisenhandels, die schon mehrfach zu ungewollten Kursausschlägen geführt hat, trägt die moderne Informationstechnik jetzt auch noch dazu bei, das Handeln der Banker von ihrer empirischen Wissensbasis zu entkoppeln. Zwischen der - nicht ausschaltbaren - Rolle mathematischer Modellierung für Transaktionen im Finanzsystem und dem fiktiven Charakter von Finanzkapital mag eine Wahlverwandtschaft bestehen. Diese Entwicklung mag durch politische Entscheidungen eingedämmt werden können - zurückzuschrauben ist sie kaum.

\section{Literatur}

Arner, Douglas W., und Michael W. Taylor. 2009. The global financial crisis and the financial stability board: Hardening the soft law of international financial regulation? University of Hong Kong, AIIFL Working Paper No. 6.

Braithwaite, John, und Peter Drahos. 2000. Global business regulation. Cambridge (UK): Cambridge University Press.

British Academy. 2009. Letter to Her Majesty the Queen, London, 22. Juli 2009. 
Busch, Andreas. 2009. Banking regulation and globalization. Oxford: Oxford University Press.

De Larosière, Jaques. 2009. High-level group on financial supervision in the EU-Report. Brüssel.

Deeg, Richard, und Susanne Lütz. 2000. Internationalization and financial federalism - The United States and Germany at the crossroads? Comparative Political Studies 33(3): 374-405.

Department of the Treasury. 2009. Financial regulatory reform: A new foundation. Rebuilding financial supervision and regulation. Washington, DC.

Draghi, Mario. 2008. A vision of a more resilient global economy. Financial Times 13. November.

FSA Financial Services Authority. 2009. The turner review. A regulatory response to the global banking crisis. London, März 2009.

G20. 2009. The global plan for recovery and reform. Final Communique, G20 London Summit, London, April 2009.

James, Harold. 2009. Die Krise der Finanzmärkte und die Rückkehr des Staates. Berlin-Brandenburgische Akademie der Wissenschaften, Berichte und Abhandlungen 15. Berlin: Akademieverlag, 23-38.

Lütz, Susanne. 2009. Geld regiert die Welt, oder: Wer steuert die Globalisierung der Finanzmärkte? In Wer regiert die Welt und mit welchem Recht? Beiträge zur Global Governance-Forschung, Hrsg. Volker Rittberger, 73-95. Baden-Baden: Nomos.

Major, Aaron. 2009. Markets and knowledge: A review of Robert Shiller's The Subprime Solution and George Soros's The New Paradigm for Financial Markets. Socio-Economic Review 7(2): 369-374.

Mayes, David G., und Geoffrey E. Wood. (Hrsg.). 2007. The structure of financial regulation. London: Routledge.

Mayntz, Renate. 2009 (2007). Die Handlungsfähigkeit des Nationalstaats in Zeiten der Globalisierung. In Über Governance. Institutionen und Prozesse politischer Regelung, Hrsg. Renate Mayntz, 53-64. Frankfurt a.M.: Campus.

Pado-Schioppa, Tommaso. 2003. Financial supervision: inside or outside central banks? In Financial Supervision in Europe, Hrsg. J.J.M. Kremers, D. Schoenmakers, und P.J. Wierts, 160-175. Cheltenham: Edward Elgar.

Posner, Elliot. 2009. Making rules for global finance: Transatlantic regulatory cooperation at the turn of the millenium. International Organization 63 (Fall 2009): 665-699.

Quaglia, Lucia. 2007. The politics of financial services regulation and supervision reform in the European Union. European Journal of Political Research 46(2): 269-290.

Reifner, Udo. 2010. Die Geldgesellschaft. Wiesbaden: VS Verlag für Sozialwissenschaften.

Reinhart, Carmen M., und Kenneth S. Rogoff. 2009. This time is different. Eight centuries of financial folly. Princeton: Princeton University Press.

Schuppert, Gunnar Folke. 2009. Staat als Prozess. Eine staatstheoretische Skizze in sieben Aufzügen. Frankfurt a.M.: Campus.

Strange, Susan. 1988. States and markets. An introduction to international political economy. London: Pinter.

The Warwick Commission on International Financial Reform: In praise of unlevel playing fields. The report of the second Warwick Commission, 2009. The University of Warwick.

Underhill, Geoffrey R.D. 1991. Markets beyond politics? The state and the internationalisation of financial markets. European Journal of Political Research 19(2-3): 197-225. 